\title{
Healthcare Cost and Utilization Associated with Biologic Treatment Patterns Among Patients with Psoriatic Arthritis: Analyses from a Large US Claims Database
}

\author{
Peter Hur ${ }^{1}$ Nina $\mathrm{Kim}^{2,3}$ • Dong Dai ${ }^{1}$. Olivia W. Piao ${ }^{4} \cdot$ Josh Z. Zheng ${ }^{4} \cdot$ Esther Yi $^{1}$
}

Accepted: 24 October 2020 / Published online: 12 November 2020

(c) The Author(s) 2020

\begin{abstract}
Background Costs associated with biologic switching and discontinuation can be high in psoriatic arthritis (PsA), and their inappropriate use may have cost implications for patients, healthcare professionals, and payers.

Objective To compare direct costs of treatment switchers, non-switchers, and discontinuers among patients with PsA who newly initiated a biologic.

Methods Patients with PsA aged $\geq 18$ years with $\geq 1$ pharmacy claim for an FDA-approved subcutaneous biologic from 1 January 2016 to 31 December 2016 were identified from the Truven Health MarketScan Databases. Patients were categorized into three mutually exclusive groups of non-switchers, switchers, and discontinuers, and healthcare costs and utilization during 1-year follow-up were described across the three groups separately.

Results A total of 2560 patients with PsA newly initiating a biologic were categorized as non-switchers (54.8\%), switchers (18.5\%), and discontinuers (26.7\%). During 1-year follow-up, after adjusting for age, sex, full-time work status, and co-morbidities, switchers had higher mean total all-cause healthcare costs than non-switchers (US $\$ 80,380$ vs. US $\$ 69,031$ ), driven by increased pharmacy (US\$66,531 vs. US $\$ 56,674$ ) and outpatient (US $\$ 10,881$ vs. US $\$ 8,235$ ) costs (all $P<0.0001$ ). Discontinuers had the lowest mean total all-cause healthcare costs (US\$50,054) but the highest medical costs (US\$20,323). Switchers and discontinuers had higher all-cause healthcare utilization than non-switchers during 1-year follow-up, except switchers had fewer hospitalizations.

Conclusions Patients with PsA who switch or discontinue biologics have higher medical costs and healthcare utilization than those continuing the same biologic. These findings highlight that discontinuing or switching biologic therapies is associated with higher costs in patients with PsA, which may inform treatment and/or formulary decision-making.
\end{abstract}

Peter Hur

peter.hur@novartis.com

1 Novartis Pharmaceuticals Corporation, One Health Plaza, East Hanover, NJ 07936-1080, USA

2 University of Texas at Austin, Austin, TX, USA

3 Baylor Scott \& White Health, Temple, TX, USA

4 KMK Consulting, Inc, East Hanover, NJ, USA

\section{Key Points}

Within 1 year of initiating a subcutaneous biologic, $54.8 \%$ of patients with PsA remained on their index biologic, $18.5 \%$ switched from their index biologic to a new therapy, and $26.7 \%$ discontinued their index biologic.

Compared with non-switchers, switchers had higher total all-cause healthcare costs, which were driven by increased prescription and medical costs; discontinuers had lower all-cause healthcare costs but the highest medical costs, potentially due to active disease.

Switchers and discontinuers had increased healthcare resource utilization (outpatient visits) than non-switchers. 


\section{Introduction}

Psoriatic arthritis (PsA) is a chronic, progressive inflammatory disease characterized by manifestations such as nail and skin changes, peripheral joint inflammation, enthesitis, dactylitis, and/or axial disorder [1], with an estimated prevalence of $0.1-0.25 \%$ in the USA [2]. Manifestations of PsA may occur alone or in combination with each other and may range from mild to severe [3]. The dual skin and musculoskeletal manifestations as well as the high association with co-morbidities complicate disease management, resulting in impairment of quality of life and psychological and physical function, and increased economic burden [4]. PsA is associated with significant direct [i.e., medical (including inpatient and outpatient) and pharmacy] and indirect (i.e., loss of work productivity) costs, which may vary depending on disease severity or additional co-morbidity burden [4-6]. In particular, patients with PsA have a significantly greater co-morbidity burden and direct healthcare costs than individuals without psoriatic disease, highlighting the need for effective treatment and improved healthcare delivery systems [7].

Biologic therapies with a stepwise approach to treating symptoms are recommended for patients who have active PsA despite conventional therapies [8-10]. Currently approved biologics for the treatment of PsA include tumor necrosis factor inhibitors (TNFis) adalimumab [11], certolizumab pegol [12], etanercept [13], golimumab [14, 15], and infliximab [16]; the interleukin (IL)-12/23 inhibitor ustekinumab $[17,18]$; the IL-17A inhibitors secukinumab [19-21], and ixekizumab [22, 23]; the selective T-cell costimulatory modulator abatacept $[24,25]$; and the IL-23 inhibitor guselkumab [26, 27]. Biologics have revolutionized PsA treatment in patients with inadequate responses to conventional therapies; however, biologics are costly contributors to direct healthcare costs. Additionally, patients may switch and/or discontinue therapies due to primary or secondary loss of efficacy, adverse events, or other reasons $[28,29]$. Therefore, it is important to understand the associations between switching or discontinuing biologics and clinical and economic impact of switching or discontinuing biologics. A few retrospective studies have been conducted in the USA using claims databases to characterize switching patterns and the associated healthcare costs and utilization in patients with PsA; however, these studies focused only on switching between TNFis [30, 31].

Due to the expansion of the biologic landscape, there is a need for a more comprehensive evaluation of the healthcare costs and utilization associated with treatment switching patterns in patients who have initiated any of the US Food and Drug Administration (FDA)-approved biologic treatments for PsA. Such an evaluation would allow for the inclusion of a larger patient population, resulting in clinically meaningful, detailed analyses to be conducted. This study aimed to compare healthcare costs and utilization between patients with PsA who switched or discontinued biologics and those who remained on the index biologic through 1 year of follow-up.

\section{Patients and Methods}

\subsection{Study Design and Patient Population}

A retrospective, noninterventional, observational cohort study was conducted using the Truven Analytics MarketScan ${ }^{\circledR}$ Commercial Claims and Encounters (CCAE) database and the Medicare Supplemental and Coordination of Benefits (MDCR) database from 1 January 2015 to 31 December 2017. Truven Analytics MarketScan is a widely used data source for analysis of epidemiologic and economic outcomes in the US healthcare system, containing claims information from more than 300 employers and 26 health plans, with more than 41.1 million covered individuals in the most recent full data year. The CCAE database contains administrative claims data from a privately insured population, provided under a variety of fee-for-service, fully capitated, and partially capitated health plans. These plans include preferred provider organizations and exclusive provider organizations, point-of-service plans, indemnity plans, health maintenance organizations, and consumer-directed health plans. The MDCR database contains administrative claims data from retirees with Medicare supplemental insurance paid by employers, employer-paid portion, services provided under Medicare-covered payment, and any out-ofpocket expenses. The CCAE and MDCR databases include fully adjudicated de-identified medical claims linked to outpatient prescription drug claims and person-level enrollment data through the use of unique patient identifiers.

Demographic variables (age, sex, employment status, insurance plan type, and geographic location) and detailed healthcare costs and utilization for healthcare services performed in both inpatient and outpatient settings were captured.

Patients aged $\geq 18$ years with $\geq 1$ pharmacy claim for an FDA-approved subcutaneously administered biologic (adalimumab, certolizumab pegol, etanercept, golimumab, secukinumab, or ustekinumab) from 1 January 2016 to 31 December 2016 (identification period) were identified in the CCAE and MDCR databases. Biologics that were approved after this identification period (abatacept, ixekizumab, and guselkumab) and/or that are administered intravenously (infliximab, abatacept) were excluded from the analysis. Eligible patients at the time of biologic initiation (index date) were continuously enrolled, had medical and pharmacy claims $\geq$ 
1 year before (baseline period) and $\geq 1$ year after the index date (follow-up period), and had $\geq 1$ PsA diagnosis [International Classification of Diseases, Ninth Revision, Clinical Modification (ICD-9-CM) 696.0 or ICD-10-CM L40.5x] during the baseline period. Patients were excluded from this study if they had other diagnoses for which biologics are also indicated and/or pharmacy claims for the index biologic during the baseline period. Additionally, the treatment washout period of 1 year allowed for a reduction in bias introduced by prevalent biologic use.

Use of the databases for health services research complies with the Health Insurance Portability and Accountability Act and federal guidance on Public Welfare and the Protection of Human Subjects.

\subsection{Study Variables}

Patients were categorized into three mutually exclusive groups based on biologic treatment patterns during the 1-year follow-up period:

- Non-switchers: Patients who remained on the index biologic with no gaps in treatment ( $>120$ days) and no other claims for biologic therapies during the 1-year follow-up period. A gap of $>120$ days was chosen to ensure that patients who stopped treatment did not restart treatment within a short time period and to accommodate ustekinumab's every-12-week dosing.

- Switchers: Patients who had $\geq$ one prescription claim for a different biologic therapy (adalimumab, certolizumab pegol, etanercept, golimumab, secukinumab, or ustekinumab) than the index biologic and who switched therapies before permissible treatment gaps ( $>120$ days) were reached during the 1-year follow-up period.

- Discontinuers: Patients who had prescription claim gaps of $>120$ days (time from exhaustion of previous days' supply) during the 1-year follow-up period.

Patient demographics (age, sex, employment status, insurance plan type, and geographic location) were assessed at the index date, and all-cause healthcare costs [including medical (inpatient, outpatient, and ER costs) and pharmacy costs] and all-cause healthcare resource utilization [hospitalizations, emergency room (ER) visits, and outpatient visits] were measured during both the 1-year baseline period and the 1-year follow-up period. All-cause healthcare costs and healthcare resource utilization were calculated per patient per year; healthcare costs were inflated to year 2017 costs (US dollars).

\subsection{Data Analysis}

Descriptive statistics were tabulated for patient characteristics and outcome variables (healthcare resource costs and utilization) comparing non-switchers versus switchers and non-switchers versus discontinuers. Continuous variables were summarized as means and SDs, whereas categorical variables were presented as counts and percentages, with missing data considered a separate category. Comparisons between treatment pattern groups were made using $\chi^{2}$ tests for categorical variables and Wilcoxon rank-sum tests or unequal variance two-sample $t$ tests for continuous variables.

Multivariable models were fitted to compare all-cause healthcare resource utilization and costs between switchers versus non-switchers and discontinuers versus non-switchers using a negative binomial distribution with log link function for all outcomes. Adjusted healthcare utilization and costs were estimated for non-switchers, switchers, and discontinuers based on the models. All models were adjusted to control for baseline co-variates, including age, sex, full-time work status (yes or no), and baseline Charlson co-morbidity index (Deyo's modification). All analyses were done using SAS ${ }^{\circledR}$ Software Version 9.4 (SAS Institute, Cary, NC, USA) in the EVICO platform.

\section{Results}

\subsection{Study Population and Patient Demographics}

This study included 94,753 patients with $\geq$ one claim for adalimumab, certolizumab pegol, etanercept, golimumab, secukinumab, or ustekinumab during the identification period between 1 January 2016 and 31 December 2016 (Fig. 1). After inclusion and exclusion criteria were applied, the final study cohort consisted of 2,560 patients with PsA who initiated a biologic; the patients were then categorized as switchers (18.5\%), non-switchers (54.8\%), or discontinuers $(26.7 \%)$. Overall, 1,123 patients $(43.9 \%)$ initiated adalimumab, 134 (5.2\%) certolizumab pegol, 660 (25.8\%) etanercept, 74 (2.9\%) golimumab, 290 (11.3\%) secukinumab, and 279 (10.9\%) ustekinumab.

Patient demographics were mostly similar across treatment groups (Table 1). Overall, the mean (SD) age was 50.5 (11.2) years; $57.7 \%$ were women, and most were from the southern region of the USA (48.4\%). Switchers and discontinuers had a higher proportion of women compared with non-switchers $(63.2 \%$ and $65.2 \%$, respectively, vs. $52.1 \%$; both $P<0.05$ ). 


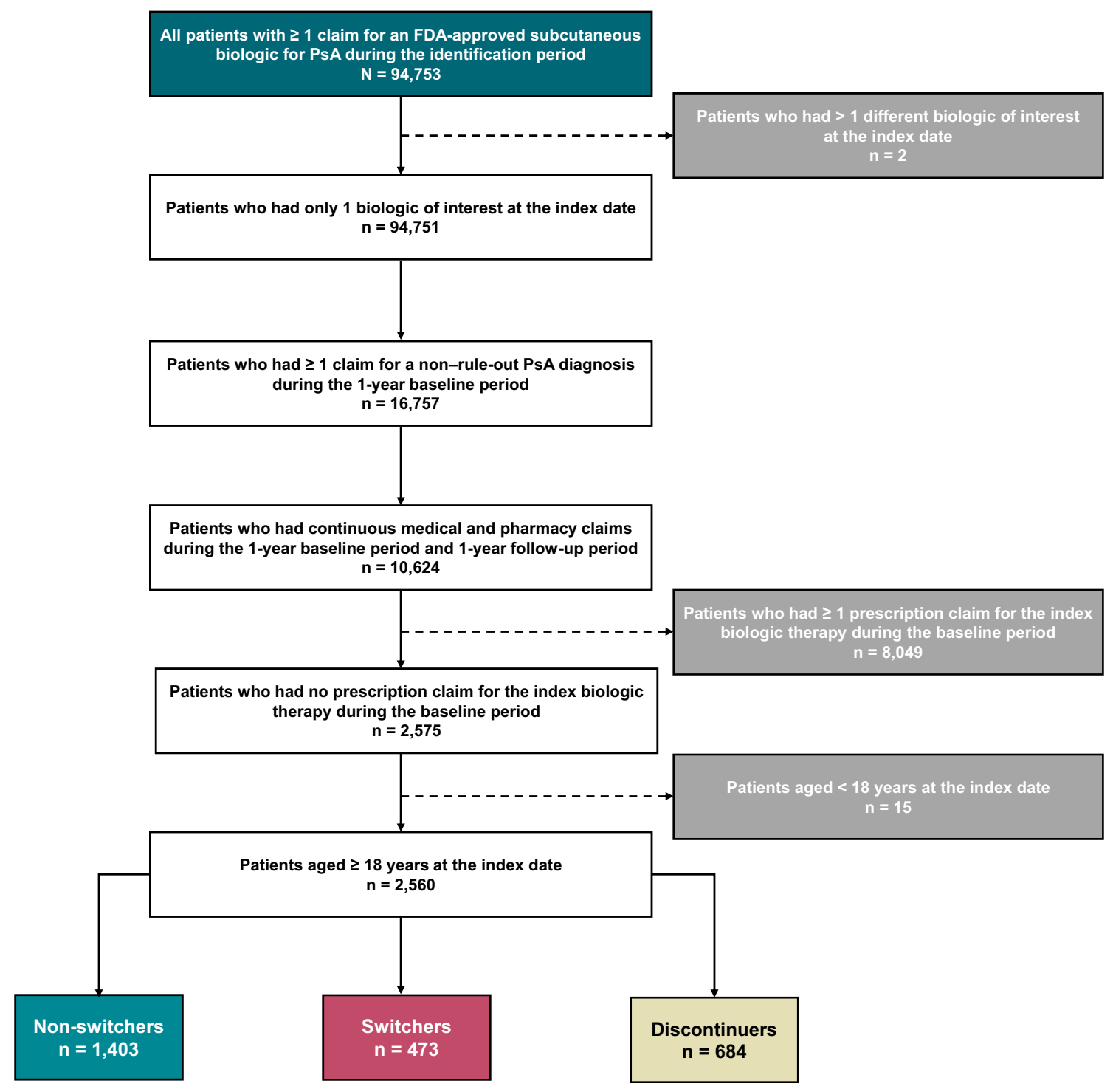

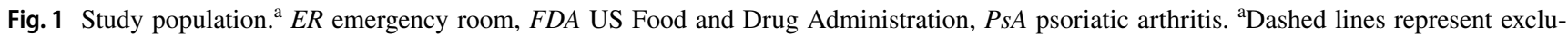
sion criteria

\subsection{Baseline All-Cause Healthcare Resource Costs and Utilization}

Across all treatment groups, the mean (SD) number of patient visits per year was $0.12(0.50)$ for hospitalizations, 0.51 (2.75) for ER visits, and 28.60 (22.21) for outpatient visits (Table 2). Discontinuers had a significantly higher mean (SD) number of ER visits per patient per year compared with non-switchers [0.81 (5.07) vs. $0.38(0.90) ; P<$ 0.05]. Both switchers and discontinuers had a significantly higher mean (SD) number of outpatient visits per patient per year than non-switchers $[29.93(20.91)$ and 30.28 (23.17), respectively, vs. 27.32 (22.10); both $P<0.05]$.
At baseline, the overall mean (SD) total all-cause healthcare cost across all treatment pattern groups was US\$27,021 (US\$42,199), with mean (SD) pharmacy and medical costs of US\$11,509 (US\$20,640) and US\$15,512 (US\$34,797), respectively (Table 2). Switchers had significantly higher mean (SD) all-cause total healthcare costs than non-switchers [US\$30,514 (US\$38,630) vs. US\$24,652 (US\$32,281); $P<0.0001]$. Switchers had slightly but significantly higher medical costs than non-switchers [US $\$ 14,883$ (US\$25,202) vs. US\$14,086 (US\$27,272); $P<0.01$ ] due to higher outpatient costs [US\$12,430 (US\$22,263) vs. US $\$ 10,268$ (US\$18,403); $P<0.001$ ] and pharmacy costs [US\$15,631 (US\$24,766) vs. US\$10,566 (US\$15,771); $P<$ 0.0001]. Discontinuers also had higher mean (SD) all-cause 
Table 1 Baseline patient demographics assessed at the index date
Table 2 Healthcare costs and resource utilization assessed during the 1-year baseline period

\begin{tabular}{lllll}
\hline Characteristic & $\begin{array}{l}\text { Overall } \\
N=2560\end{array}$ & $\begin{array}{l}\text { Non-switchers } \\
n=1403\end{array}$ & $\begin{array}{l}\text { Switchers } \\
n=473\end{array}$ & $\begin{array}{l}\text { Discontinuers } \\
n=684\end{array}$ \\
\hline Age, mean (SD), years & $50.5(11.2)$ & $50.5(11.1)$ & $50.5(10.8)$ & $50.3(11.6)$ \\
Female, $n$ (\%) & $1476(57.7)$ & $731(52.1)$ & $299(63.2)^{\mathrm{a}}$ & $446(65.2)^{\mathrm{a}}$ \\
Insurance plan type, $n(\%)$ & & & & \\
Fee for service & $2272(88.8)$ & $1239(88.3)$ & $420(88.8)$ & $613(89.6)$ \\
HMO and POS capitation & $267(10.4)$ & $154(11.0)$ & $46(9.7)$ & $67(9.8)$ \\
Unknown & $21(0.8)$ & $10(0.7)$ & $7(1.5)$ & $4(0.6)$ \\
US region, $n$ (\%) & & & & \\
South & $1239(48.4)$ & $666(47.5)$ & $247(52.2)$ & $326(47.7)$ \\
North Central & $450(17.6)$ & $248(17.7)$ & $82(17.3)$ & $120(17.5)$ \\
Northeast & $536(20.9)$ & $298(21.2)$ & $94(19.9)$ & $144(21.1)$ \\
West & $334(13.0)$ & $191(13.6)$ & $50(10.6)$ & $93(13.6)$ \\
Unknown & $1(0.0)$ & 0 & 0 & $1(0.1)$ \\
Employment status, $n(\%)$ & & & & \\
Active full time & $1743(68.1)$ & $981(69.9)$ & $318(67.2)$ & $444(64.9)$ \\
Active part time or seasonal & $33(1.3)$ & $18(1.3)$ & $8(1.7)$ & $7(1.0)$ \\
Early retiree & $151(5.9)$ & $80(5.7)$ & $25(5.3)$ & $46(6.7)$ \\
Medicare eligible retiree & $155(6.1)$ & $83(5.9)$ & $29(6.1)$ & $43(6.3)$ \\
Retiree (status unknown) & 0 & 0 & 0 & 0 \\
COBRA continuee & $10(0.4)$ & $8(0.6)$ & $1(0.2)$ & $1(0.1)$ \\
Long-term disability & $17(0.7)$ & $7(0.5)$ & $5(1.1)$ & $5(0.7)$ \\
Surviving spouse/dependent & $20(0.8)$ & $12(0.9)$ & 0 & $8(1.2)$ \\
Other/unknown & $431(16.8)$ & $214(15.3)$ & $87(18.4)$ & $130(19.0)$ \\
\hline
\end{tabular}

$H M O$ health maintenance organization, $P O S$ point of service ${ }^{\text {a }} P<0.05$ vs. non-switchers

\begin{tabular}{lllll}
\hline Characteristic & $\begin{array}{l}\text { Overall } \\
N=2560\end{array}$ & $\begin{array}{l}\text { Non-switchers } \\
n=1403\end{array}$ & $\begin{array}{l}\text { Switchers } \\
n=473\end{array}$ & $\begin{array}{l}\text { Discontinuers } \\
n=684\end{array}$ \\
\hline Total all-cause healthcare & 27,021 & 24,652 & 30,514 & 29,463 \\
costs, mean (SD), $\$^{\mathrm{a}}$ & $(42,199)$ & $(32,281)$ & $(38,630)^{\mathrm{b}}$ & $(58,955)$ \\
Pharmacy costs & 11,509 & 10,566 & 15,631 & 10,593 \\
& $(20,640)$ & $(15,771)$ & $(24,766)^{\mathrm{b}}$ & $(25,440)$ \\
Medical costs & 15,512 & 14,086 & 14,883 & 18,871 \\
& $(34,797)$ & $(27,272)$ & $(25,202)^{\mathrm{b}}$ & $(50,549)^{\mathrm{b}}$ \\
ER costs & 913 & 726 & 845 & 1342 \\
& $(4,313)$ & $(3,054)$ & $(2,204)$ & $(6,851)^{\mathrm{b}}$ \\
Inpatient costs & 3445 & 3091 & 1608 & 5440 \\
& $(24,242)$ & $(15,566)$ & $(8,676)$ & $(40,569)$ \\
Outpatient costs & 11,154 & 10,268 & 12,430 & 12,089 \\
& $(19,280)$ & $(18,403)$ & $(22,263)^{\mathrm{b}}$ & $(18,745)^{\mathrm{b}}$ \\
Total all-cause healthcare resource utilization & per patient per year, mean $(\mathrm{SD}), n$ & \\
Hospitalizations & $0.12(0.50)$ & $0.11(0.40)$ & $0.09(0.37)$ & $0.16(0.71)$ \\
ER visits & $0.51(2.75)$ & $0.38(0.90)$ & $0.47(1.05)$ & $0.81(5.07)^{\mathrm{b}}$ \\
Outpatient visits & $28.60(22.21)$ & $27.32(22.10)$ & $29.93(20.91)^{\mathrm{b}}$ & $30.28(23.17)^{\mathrm{b}}$ \\
\hline
\end{tabular}

ER emergency room

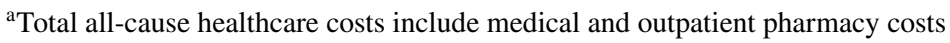

${ }^{\mathrm{b}} P<0.05$ vs. non-switchers

${ }^{\mathrm{c}}$ Medical costs include ER, inpatient, and outpatient costs 
total healthcare costs than non-switchers [US $\$ 29,463$ (US\$58,955)], but the difference was not significant ( $P$ $=0.49$ ). However, discontinuers had significantly higher medical costs than non-switchers [US\$18,871 (US\$50,549)] due to higher ER [US\$1,342 (US\$6,851) vs. US $\$ 726$ (US\$3,054); $P=0.0012$ ] and outpatient [US $\$ 12,089$ (US $\$ 18,745)$ vs. US $\$ 10,268$ (US $\$ 18,403) ; P<0.01$ ] costs.

\subsection{Unadjusted All-Cause Healthcare Resource Costs and Utilization During the 1-Year Follow-Up Period}

In the 1-year follow-up period, switchers had a statistically significantly higher total all-cause healthcare cost compared with non-switchers, whereas discontinuers had a lower total all-cause healthcare cost (Table 3). Switchers had a higher mean (SD) total all-cause healthcare cost compared with non-switchers [US\$80,411 (US\$50,845) vs. US\$69,152 (US\$34,699); $P<0.0001$ ], which was contributed to by increased medical [US\$13,898 (US\$39,400) vs. US\$12,583 (US\$29,057); $P<0.0001]$ and pharmacy [US\$66,513 (US\$30,455) vs. US\$56,570 (US\$24,006); $P<$ $0.0001]$ costs. The mean total all-cause healthcare cost for discontinuers [US $\$ 52,708$ (US\$66,702); $P<0.0001$ vs. nonswitchers] was much lower than that for the other treatment pattern groups due to reduced pharmacy costs [US $\$ 28,826$ (US\$23,771); $P<0.0001$ vs. non-switchers]; however, discontinuers had the highest medical costs [US $\$ 23,882$ (US\$62,440); $P<0.0001]$ vs. non-switchers.

In the 1-year follow-up period, switchers and discontinuers had higher all-cause healthcare utilization than nonswitchers (Table 3). Switchers had a significantly higher mean (SD) number of outpatient visits per patient per year compared with non-switchers [30.60 (21.52) vs. 25.04
(20.14); $P<0.0001]$. Discontinuers had a significantly higher mean (SD) number of hospitalizations [0.22 (0.75) vs. $0.10(0.42) ; P<0.0001]$ and ER [0.88 (5.18) vs. 0.36 (1.02); $P<0.0001]$ and outpatient visits [30.13 (25.03) vs. 25.04 (20.14); $P<0.0001]$ per patient per year compared with non-switchers.

\subsection{Adjusted All-Cause Healthcare Resource Costs and Utilization During the 1-Year Follow-Up Period}

Multivariable modeling adjusted for co-variates (age, sex, fulltime employment, and Charlson co-morbidity index scores) further showed the healthcare cost disparities between switchers and discontinuers versus non-switchers during the 1-year follow-up period (Table 4). Similar to the unadjusted analysis, switchers had significantly higher total all-cause healthcare costs than non-switchers, which was driven by significantly higher pharmacy costs and outpatient costs (all $P<0.0001$ vs. non-switchers). Discontinuers continued to have significantly lower total all-cause healthcare costs than non-switchers, largely due to significantly lower pharmacy costs (both $P<$ 0.0001 vs. non-switchers). However, after adjusting for baseline co-variates that may be associated with a worse health state (e.g., age and co-morbidities), discontinuers still had significantly higher medical costs $(P<0.0001)$, including higher ER $(P=0.0187)$ and outpatient costs $(P<0.0001)$.

Similarly, differences in healthcare resource utilization among switchers and discontinuers compared with non-switchers were maintained during the 1-year followup period when adjusted for the same co-variates as above (Table 4). Compared with non-switchers, ER visits and outpatient visits were more frequent among switchers [adjusted ratio $(95 \% \mathrm{CI}), 1.31(1.01,1.71)$ and $1.20(1.12$,
Table 3 Unadjusted healthcare costs and resource utilization during 1-year follow-up

\begin{tabular}{|c|c|c|c|}
\hline Unadjusted all-cause outcomes & $\begin{array}{l}\text { Non-switchers } \\
n=1,403\end{array}$ & $\begin{array}{l}\text { Switchers } \\
n=473\end{array}$ & $\begin{array}{l}\text { Discontinuers } \\
n=684\end{array}$ \\
\hline \multicolumn{4}{|l|}{ Healthcare costs, mean (SD), \$ } \\
\hline Total all-cause healthcare costs ${ }^{\mathrm{a}}$ & $69,152(34,699)$ & $80,411(50,845)^{\mathrm{b}}$ & $52,708(66,702)^{\mathrm{b}}$ \\
\hline Pharmacy costs & $56,570(24,006)$ & $66,513(30,455)^{\mathrm{b}}$ & $28,826(23,771)^{\mathrm{b}}$ \\
\hline Medical costs & $12,583(29,057)$ & $13,898(39,400)^{\mathrm{b}}$ & $23,882(62,440)^{\mathrm{b}}$ \\
\hline Outpatient costs & $8838(17,995)$ & $11,596(37,371)^{\mathrm{b}}$ & $13,359(23,562)^{\mathrm{b}}$ \\
\hline Inpatient costs & $3064(17,749)$ & $1522(7903)$ & $9078(49,288)^{\mathrm{b}}$ \\
\hline ER costs & $680(2524)$ & $780(2407)$ & $1445(6270)^{\mathrm{b}}$ \\
\hline \multicolumn{4}{|c|}{ Healthcare utilization, per patient per year, mean (SD) } \\
\hline Hospitalizations & $0.10(0.42)$ & $0.07(0.29)$ & $0.22(0.75)^{\mathrm{b}}$ \\
\hline ER visits & $0.36(1.02)$ & $0.45(1.05)$ & $0.88(5.18)^{\mathrm{b}}$ \\
\hline Outpatient visits & $25.04(20.14)$ & $30.60(21.52)^{b}$ & $30.13(25.03)^{b}$ \\
\hline
\end{tabular}

ER emergency room

${ }^{\text {a }}$ Total all-cause healthcare costs include medical and outpatient pharmacy costs

${ }^{\mathrm{b}} P<0.0001$ vs. non-switchers 
Table 4 Adjusted healthcare costs and resource utilization during 1-year follow-up ${ }^{\mathrm{a}}$

\begin{tabular}{|c|c|c|c|c|c|}
\hline Adjusted all-cause outcomes ${ }^{b}$ & $\begin{array}{l}\text { Non-switchers } \\
n=1403\end{array}$ & $\begin{array}{l}\text { Switchers } \\
n=473\end{array}$ & $\begin{array}{l}\text { Discontinuers } \\
n=684\end{array}$ & $\begin{array}{l}\text { Switchers vs. } \\
\text { non-switchers }\end{array}$ & $\begin{array}{l}\text { Discontinuers vs. } \\
\text { non-switchers }\end{array}$ \\
\hline Healthcare costs, $\$$ & $\operatorname{LSM}(95 \% \mathrm{CI})$ & & & Ratio $(95 \%$ CI) & \\
\hline Total healthcare costs & $\begin{array}{l}69,031 \\
(67,174,70,939)\end{array}$ & $\begin{array}{l}80,380 \\
(76,705,84,230)\end{array}$ & $\begin{array}{l}50,054 \\
(48,131,52,054)\end{array}$ & $1.16(1.10,1.23)^{\mathrm{c}}$ & $0.73(0.69,0.76)^{\mathrm{c}}$ \\
\hline Pharmacy costs & $\begin{array}{l}56,674 \\
(54,920,58,485)\end{array}$ & $\begin{array}{l}66,531 \\
(63,034,70,222)\end{array}$ & $\begin{array}{l}28,533 \\
(27,273,29,850)\end{array}$ & $1.17(1.10,1.25)^{\mathrm{c}}$ & $0.50(0.48,0.53)^{\mathrm{c}}$ \\
\hline Medical costs & $\begin{array}{l}11,444 \\
(10,685,12,257)\end{array}$ & $\begin{array}{l}13,018 \\
(11,573,14,642)\end{array}$ & $\begin{array}{l}20,323 \\
(18,418,22,424)\end{array}$ & $1.14(0.99,1.30)$ & $1.78(1.57,2.00)^{\mathrm{c}}$ \\
\hline Outpatient costs & $\begin{array}{l}8235 \\
(7725,8778)\end{array}$ & $\begin{array}{l}10,881 \\
(9749,12,143)\end{array}$ & $\begin{array}{l}12,288 \\
(11,211,13,469)\end{array}$ & $1.32(1.16,1.50)^{\mathrm{c}}$ & $1.49(1.33,1.67)^{\mathrm{c}}$ \\
\hline Inpatient costs & $\begin{array}{l}2418 \\
(1309,4466)\end{array}$ & $1310(456,3765)$ & $\begin{array}{l}5551 \\
(2302,13,387)\end{array}$ & $0.54(0.16,1.86)$ & $2.30(0.77,6.81)$ \\
\hline ER costs & $656(476,903)$ & $760(440,1314)$ & $1292(816,2047)$ & $1.16(0.61,2.19)$ & $1.97(1.12,3.47)^{\mathrm{d}}$ \\
\hline \multicolumn{6}{|l|}{ Healthcare resource utilization } \\
\hline Hospitalizations & $0.09(0.07,0.11)$ & $0.06(0.04,0.09)$ & $0.19(0.15,0.24)$ & $0.74(0.48,1.16)$ & $2.18(1.59,2.98)^{\mathrm{c}}$ \\
\hline ER visits & $0.33(0.29,0.38)$ & $0.43(0.35,0.55)$ & $0.75(0.63,0.89)$ & $1.31(1.01,1.71)^{\mathrm{d}}$ & $2.27(1.82,2.83)^{\mathrm{c}}$ \\
\hline Outpatient visits & $\begin{array}{l}24.53 \\
(23.67,25.42)\end{array}$ & $\begin{array}{l}29.40 \\
(27.67,31.24)\end{array}$ & $\begin{array}{l}27.88 \\
(26.50,29.33)\end{array}$ & $1.20(1.12,1.29)^{\mathrm{c}}$ & $1.14(1.07,1.21)^{\mathrm{c}}$ \\
\hline
\end{tabular}

ER emergency room, LSM least squares means

${ }^{a}$ Covariates included age, sex, full-time employment (yes or no), and baseline Charlson co-morbidity index

${ }^{\mathrm{b}}$ All outcomes were derived from a negative binomial distribution with $\log$ link functions for models

${ }^{\mathrm{c}} P<0.0001$ vs. non-switchers

${ }^{\mathrm{d}} P<0.05$ vs. non-switchers

1.29), respectively; $P<0.05$ and $P<0.0001$, respectively, vs. non-switchers]. All healthcare resource utilization categories remained significantly higher among discontinuers compared with non-switchers when controlling for baseline co-variates, with adjusted ratios (95\% CIs) of 2.18 (1.59, 2.98) for hospitalizations, 2.27 (1.82, 2.83) for ER visits, and $1.14(1.07,1.21)$ for outpatient visits (all $P<0.0001$ vs. non-switchers).

\section{Discussion}

In this retrospective analysis of US administrative claims databases, patients with PsA who switched biologics endured a greater economic burden than those who remained on the index biologic. During the 1-year follow-up period, both discontinuers and switchers had higher frequencies of ER and outpatient visits than non-switchers; furthermore, discontinuers had the highest incidence of inpatient visits. Patients who switched biologics had the highest total allcause healthcare costs during the 1-year follow-up, contributed to by an increase in both pharmacy and medical costs.

Our results suggest that patients who remained on a biologic experienced a decreased economic burden, because those who switched or discontinued biologics had higher medical costs and increased healthcare resource utilization, while patients who switched also had higher pharmacy costs. Overall, $54.8 \%$ of patients remained on the index biologic, $18.5 \%$ switched the biologic, and $26.7 \%$ discontinued the biologic therapy within the 1-year follow-up period; these results are similar to those from previous studies using the Humana US claims database to evaluate switching patterns across multiple immune-mediated diseases, including rheumatoid arthritis, ankylosing spondylitis, psoriasis, and PsA [30], and in the Truven databases among patients with PsA [32]. From the Humana US claims database, Howe and colleagues reported that both new patients and continuing patients had similar mean annual costs per treated patient; however, continuing patients had the highest medical costs, whereas new patients had the highest pharmacy costs [30]. Additionally, from the US Truven Analytics MarketScan database, Palmer and colleagues showed that although patients with PsA who remained on the first-line TNFi incurred higher medical costs than those switching to a second- or third-line TNFi, pharmacy costs increased with each new line of therapy [31]. In both analyses, studies only examined the healthcare costs associated with switching between TNFis. Our study is more reflective of treatment patterns observed in current clinical practice as it includes newer biologic agents with different mechanisms of action (e.g., secukinumab and ustekinumab). Furthermore, our study included more recent data (2015-2017) compared with 
the previous studies from the Humana [30] (2008-2011) and Truven [31] (2005-2013) databases. These differences may suggest changes in clinical practice over time with increased treatment choices. Of note, patients with PsA who continued on the index biologic had higher rates of persistence on index therapy than new initiators [30]. Although persistence was not evaluated in our study, our study demonstrated that switching or discontinuing biologics leads to significant economic burdens and highlights the importance of choosing the proper initial therapy.

During the baseline period, switchers had the highest total all-cause healthcare costs among the three treatment pattern groups. The higher total all-cause healthcare costs for switchers were contributed to by both higher medical and pharmacy costs compared with those for non-switchers, supporting previous studies suggesting that switchers may have more severe disease at baseline [28, 29]. Switchers had the highest pharmacy costs at baseline and during 1-year follow-up, while non-switchers and discontinuers had similar pharmacy costs during the baseline period. The increase in pharmacy costs during the baseline period may be partially due to general behavioral patterns of switchers, who may favor switching between multiple medications quickly. Additionally, at baseline and during 1-year follow-up, discontinuers had the highest mean medical costs, implying that these patients may have more severe, untreated disease than those who remain on the same biologic or switch to other biologics and would have benefited from earlier biologic treatment. These findings were reaffirmed by adjusted analyses controlling for baseline co-variates that may have contributed to a worse clinical state (e.g., age and co-morbidities).

It has been shown in a combined cohort of patients with PsA, psoriasis, and rheumatoid arthritis that younger age, female sex, higher out-of-pocket costs, greater disease severity, and more co-morbidities are associated with lower adherence to biologics [33]. Interestingly, approximately two-thirds of discontinuers or switchers in our study were women compared with approximately 50\% of non-switchers. This increased female predominance has been previously reported among TNFi discontinuers [34-36]. Female sex has been associated with increased drug discontinuation or premature treatment termination compared with male sex, which may be attributed to inferior response or additional adverse effects [34, 37].

Furthermore, of interest to payers, discontinuing biologics leads to medication wastage because initiating a costly drug and not completing treatment results in excessive, improper use of medication and an unnecessary financial burden. Instances of wasted resources have been documented and analyzed in studies involving treatment nonadherence and discontinuation in other disease models $[38,39]$. From a policy and formulary perspective, this study also informs administrators on the costs of switching and/or discontinuing therapies, allowing them to compare incurring costs when patients and physicians switch therapies versus the cost savings of having patients continue the current therapy.

Because there are only a few studies in the USA that have reported on healthcare costs and utilization associated with biologic switching or discontinuation among patients with PsA, these findings provide payers and healthcare providers with insights on the financial implications of switching, suggesting better management of treatment and proper formulary decisions to mitigate both the clinical and the economic burdens of PsA. Future studies are needed to investigate clinical outcomes associated with treatment patterns and costs of individual therapies, perhaps by linking medical and pharmacy claims to the patients' electronic medical records.

Despite having several strengths, this study has a few limitations due to the nature of the study design and data source. Because all data were dependent on claims information, there is potential for misclassification of clinical characteristics or events. Furthermore, the validity of the analysis related to switching is only sensitive to the accuracy of the data recorded from the claims database. Although the measurement error around claims for expensive medication is likely low, uncertainty around the cost of switching may remain. Because the data are limited to patients who have commercial and Medicare supplemental insurance, the current findings may not be generalizable to the general population of patients with PsA; additionally, costs may have been underestimated for patients who received supplemental healthcare or those who did not have insurance coverage and were not included in the claims database. Based on the definition of a permissible treatment gap of $>120$ days, patients may be misclassified as discontinuers, which may bias differences in cost estimates toward the null. Additional analyses using a more recent time frame to capture newer biologics and linking claims to data from electronic medical records are needed to provide additional clinical information that are lacking in claims databases. Long-term analyses of real-world data would also help to substantiate these findings.

\section{Conclusions}

This analysis of US administrative claims data suggests that patients with PsA who switch or discontinue biologics within the first year of initiating a biologic have higher medical costs and healthcare utilization than those continuing the same biologic. Higher mean total all-cause healthcare costs among switchers versus non-switchers was primarily driven by increased pharmacy costs. These findings highlight that discontinuing or switching biologic 
therapies is associated with higher healthcare costs and resource utilization in patients with PsA, which may inform treatment and/or formulary decision-making. Overall, these results suggest a need for additional studies to evaluate the potential clinical impact of patient treatmentswitching behavioral patterns and to identify treatments to mitigate the clinical and economic burden caused by switching or discontinuing biologic therapies.

\section{Declarations}

Funding This study was sponsored by Novartis Pharmaceuticals Corporation, East Hanover, NJ, USA. Support for third-party writing assistance for this manuscript, furnished by Meaghan Paganelli, $\mathrm{PhD}$, of Health Interactions, Inc, was provided by Novartis Pharmaceuticals Corporation, East Hanover, NJ, USA.

Conflicts of Interest P. Hur, D. Dai, and E. Yi are employees of Novartis Pharmaceuticals Corporation. N. Kim was a postdoctoral fellow at the University of Texas at Austin and Baylor Scott and White Health, providing services to Novartis Pharmaceuticals Corporation, at the time of this study. O. W. Piao was an employee of KMK Consulting, Inc, at the time of this study and is a current employee of Flatiron Health. J. Z. Zheng is an employee of KMK Consulting, Inc, and works as a consultant for Novartis.

Ethics Approval Because the current study used only de-identified patient records and did not involve the collection, use, or transmittal of individually identifiable data, institutional review board approval was not required to conduct this study.

Informed Consent Not applicable; this retrospective study of claims data used de-identified patient records and did not involve the collection, use, or transmittal of individually identifiable data.

Data Availability All data generated or analyzed during this study are included in this published article.

Author Contributions All authors made substantial contributions to the conception and design, execution, or analysis and interpretation of the data; were involved in drafting and critically revising the manuscript; have given necessary attention to ensure the integrity of the work; have read and approved all versions of this manuscript and its submission; and fulfill the ICMJE guidelines for authorship.

Open Access This article is licensed under a Creative Commons Attribution-NonCommercial 4.0 International License, which permits any non-commercial use, sharing, adaptation, distribution and reproduction in any medium or format, as long as you give appropriate credit to the original author(s) and the source, provide a link to the Creative Commons licence, and indicate if changes were made. The images or other third party material in this article are included in the article's Creative Commons licence, unless indicated otherwise in a credit line to the material. If material is not included in the article's Creative Commons licence and your intended use is not permitted by statutory regulation or exceeds the permitted use, you will need to obtain permission directly from the copyright holder. To view a copy of this licence, visit http://creativecommons.org/licenses/by-nc/4.0/.

\section{References}

1. Gladman DD. Clinical features and diagnostic considerations in psoriatic arthritis. Rheum Dis Clin North Am. 2015;41:569-79.

2. Gelfand JM, Gladman DD, Mease PJ, Smith N, Margolis DJ, Nijsten T, et al. Epidemiology of psoriatic arthritis in the population of the United States. J Am Acad Dermatol. 2005;53:573.

3. Gottlieb A, Korman NJ, Gordon KB, Feldman SR, Lebwohl M, Koo JY, et al. Guidelines of care for the management of psoriasis and psoriatic arthritis: section 2. Psoriatic arthritis: overview and guidelines of care for treatment with an emphasis on the biologics. J Am Acad Dermatol. 2008;58:851-64.

4. Lee S, Mendelsohn A, Sarnes E. The burden of psoriatic arthritis: a literature review from a global health systems perspective. P T. 2010;35:680-9.

5. Poole CD, Lebmeier M, Ara R, Rafia R, Currie CJ. Estimation of health care costs as a function of disease severity in people with psoriatic arthritis in the UK. Rheumatology (Oxford). 2010;49:1949-56.

6. Kawalec P, Malinowski KP, Pilc A. Disease activity, quality of life and indirect costs of psoriatic arthritis in Poland. Rheumatol Int. 2016;36:1223-30.

7. Merola JF, Herrera V, Palmer JB. Direct healthcare costs and comorbidity burden among patients with psoriatic arthritis in the USA. Clin Rheumatol. 2018;37:2751-61.

8. Coates LC, Kavanaugh A, Mease PJ, Soriano ER, Laura Acosta-Felquer M, Armstrong AW, et al. Group for Research and Assessment of Psoriasis and Psoriatic Arthritis 2015 treatment recommendations for psoriatic arthritis. Arthritis Rheum. 2016;68:1060-71.

9. Gossec L, Smolen JS, Ramiro S, de Wit M, Cutolo M, Dougados M, et al. European League Against Rheumatism (EULAR) recommendations for the management of psoriatic arthritis with pharmacological therapies: 2015 update. Ann Rheum Dis. 2016;75:499-510.

10. Singh JA, Guyatt G, Ogdie A, Gladman DD, Deal C, Deodhar A, et al. Special Article: 2018 American College of Rheumatology/National Psoriasis Foundation Guideline for the Treatment of Psoriatic Arthritis. Arthritis Care Res (Hoboken). 2019;71:2-29.

11. Mease PJ, Gladman DD, Ritchlin CT, Ruderman EM, Steinfeld $\mathrm{SD}$, Choy EH, et al. Adalimumab for the treatment of patients with moderately to severely active psoriatic arthritis: results of a double-blind, randomized, placebo-controlled trial. Arthritis Rheum. 2005;52:3279-89.

12. Mease PJ, Fleischmann R, Deodhar AA, Wollenhaupt J, Khraishi M, Kielar D, et al. Effect of certolizumab pegol on signs and symptoms in patients with psoriatic arthritis: 24-week results of a Phase 3 double-blind randomised placebo-controlled study (RAPID-PsA). Ann Rheum Dis. 2014;73:48-55.

13. Mease PJ, Kivitz AJ, Burch FX, Siegel EL, Cohen SB, Ory P, et al. Etanercept treatment of psoriatic arthritis: safety, efficacy, and effect on disease progression. Arthritis Rheum. 2004;50:2264-72.

14. Kavanaugh A, McInnes I, Mease P, Krueger GG, Gladman D, Gomez-Reino J, et al. Golimumab, a new human tumor necrosis factor alpha antibody, administered every four weeks as a subcutaneous injection in psoriatic arthritis: twenty-four-week efficacy and safety results of a randomized, placebo-controlled study. Arthritis Rheum. 2009;60:976-86.

15. Kavanaugh A, Husni ME, Harrison DD, Kim L, Lo KH, Leu JH, et al. Safety and efficacy of intravenous golimumab in patients with active psoriatic arthritis: results through week twenty-four of the GO-VIBRANT study. Arthritis Rheum. 2017;69:2151-61.

16. Antoni C, Krueger GG, de Vlam K, Birbara C, Beutler A, Guzzo C, et al. Infliximab improves signs and symptoms of 
psoriatic arthritis: results of the IMPACT 2 trial. Ann Rheum Dis. 2005;64:1150-7.

17. McInnes IB, Kavanaugh A, Gottlieb AB, Puig L, Rahman P, Ritchlin C, et al. Efficacy and safety of ustekinumab in patients with active psoriatic arthritis: 1 year results of the phase 3, multicentre, double-blind, placebo-controlled PSUMMIT 1 trial. Lancet. 2013;382:780-9.

18. Ritchlin C, Rahman P, Kavanaugh A, McInnes IB, Puig L, Li S, et al. Efficacy and safety of the anti-IL-12/23 p40 monoclonal antibody, ustekinumab, in patients with active psoriatic arthritis despite conventional non-biological and biological anti-tumour necrosis factor therapy: 6-month and 1-year results of the phase 3 , multicentre, double-blind, placebo-controlled, randomised PSUMMIT 2 trial. Ann Rheum Dis. 2014;73:990-9.

19. McInnes IB, Sieper J, Braun J, Emery P, van der Heijde D, Isaacs JD, et al. Efficacy and safety of secukinumab, a fully human antiinterleukin-17A monoclonal antibody, in patients with moderateto-severe psoriatic arthritis: a 24-week, randomised, double-blind, placebo-controlled, phase II proof-of-concept trial. Ann Rheum Dis. 2014;73:349-56.

20. McInnes IB, Mease PJ, Kirkham B, Kavanaugh A, Ritchlin CT, Rahman P, et al. Secukinumab, a human anti-interleukin17A monoclonal antibody, in patients with psoriatic arthritis (FUTURE 2): a randomised, double-blind, placebo-controlled, phase 3 trial. Lancet. 2015;386:1137-46.

21. Mease PJ, McInnes IB, Kirkham B, Kavanaugh A, Rahman P, van der Heijde D, et al. Secukinumab inhibition of interleukin-17A in patients with psoriatic arthritis. N Engl J Med. 2015;373:1329-39.

22. Mease PJ, van der Heijde D, Ritchlin CT, Okada M, Cuchacovich RS, Shuler CL, et al. Ixekizumab, an interleukin-17A specific monoclonal antibody, for the treatment of biologic-naive patients with active psoriatic arthritis: results from the 24 -week randomised, double-blind, placebo-controlled and active (adalimumab)-controlled period of the phase III trial SPIRIT-P1. Ann Rheum Dis. 2017;76:79-87.

23. Nash P, Kirkham B, Okada M, Rahman P, Combe B, Burmester $\mathrm{GR}$, et al. Ixekizumab for the treatment of patients with active psoriatic arthritis and an inadequate response to tumour necrosis factor inhibitors: results from the 24-week randomised, doubleblind, placebo-controlled period of the SPIRIT-P2 phase 3 trial. Lancet. 2017;389:2317-27.

24. Mease P, Genovese MC, Gladstein G, Kivitz AJ, Ritchlin C, Tak PP, et al. Abatacept in the treatment of patients with psoriatic arthritis: results of a six-month, multicenter, randomized, double-blind, placebo-controlled, phase II trial. Arthritis Rheum. 2011;63:939-48.

25. Mease PJ, Gottlieb AB, van der Heijde D, FitzGerald O, Johnsen A, Nys M, et al. Efficacy and safety of abatacept, a T-cell modulator, in a randomised, double-blind, placebo-controlled, phase III study in psoriatic arthritis. Ann Rheum Dis. 2017;76:1550-8.

26. Deodhar A, Helliwell PS, Boehncke WH, Kollmeier AP, Hsia EC, Subramanian RA, et al. Guselkumab in patients with active psoriatic arthritis who were biologic-naive or had previously received TNF $\alpha$ inhibitor treatment (DISCOVER-1): a doubleblind, randomised, placebo-controlled phase 3 trial. Lancet. 2020;395:1115-25.

27. Mease PJ, Rahman P, Gottlieb AB, Kollmeier AP, Hsia EC, Xu $\mathrm{XL}$, et al. Guselkumab in biologic-naive patients with active psoriatic arthritis (DISCOVER-2): a double-blind, randomised, placebo-controlled phase 3 trial. Lancet. 2020;395:1126-36.
28. Costa L, Perricone C, Chimenti MS, Del Puente A, Caso P, Peluso $\mathrm{R}$, et al. Switching between biological treatments in psoriatic arthritis: a review of the evidence. Drugs R D. 2017;17:509-22.

29. Merola JF, Lockshin B, Mody EA. Switching biologics in the treatment of psoriatic arthritis. Semin Arthritis Rheum. 2017;47:29-37.

30. Howe A, Eyck LT, Dufour R, Shah N, Harrison DJ. Treatment patterns and annual drug costs of biologic therapies across indications from the Humana commercial database. J Manag Care Spec Pharm. 2014;20:1236-44.

31. Palmer JB, Li Y, Herrera V, Liao M, Tran M, Ozturk ZE. Treatment patterns and costs for anti-TNFalpha biologic therapy in patients with psoriatic arthritis. BMC Musculoskelet Disord. 2016;17:261.

32. Oelke KR, Chambenoit O, Majjhoo AQ, Gray S, Higgins K, Hur P. Persistence and adherence of biologics in US patients with psoriatic arthritis: analyses from a claims database. J Comp Eff Res. 2019;8:607-21.

33. Murage MJ, Tongbram V, Feldman SR, Malatestinic WN, Larmore CJ, Muram TM, et al. Medication adherence and persistence in patients with rheumatoid arthritis, psoriasis, and psoriatic arthritis: a systematic literature review. Patient Prefer Adherence. 2018;12:1483-503.

34. Iannone F, Lopriore S, Bucci R, Scioscia C, Anelli MG, Notarnicola A, et al. Two-year survival rates of anti-TNF-alpha therapy in psoriatic arthritis (PsA) patients with either polyarticular or oligoarticular PsA. Scand J Rheumatol. 2015;44:192-9.

35. Saad AA, Ashcroft DM, Watson KD, Hyrich KL, Noyce PR, Symmons DP, et al. Persistence with anti-tumour necrosis factor therapies in patients with psoriatic arthritis: observational study from the British Society of Rheumatology Biologics Register. Arthritis Res Ther. 2009;11:R52.

36. Glintborg B, Ostergaard M, Krogh NS, Andersen MD, Tarp $\mathrm{U}$, Loft AG, et al. Clinical response, drug survival, and predictors thereof among 548 patients with psoriatic arthritis who switched tumor necrosis factor alpha inhibitor therapy: results from the Danish Nationwide DANBIO Registry. Arthritis Rheum. 2013;65:1213-23.

37. Heiberg MS, Koldingsnes W, Mikkelsen K, Rodevand E, Kaufmann C, Mowinckel P, et al. The comparative one-year performance of anti-tumor necrosis factor alpha drugs in patients with rheumatoid arthritis, psoriatic arthritis, and ankylosing spondylitis: results from a longitudinal, observational, multicenter study. Arthritis Rheum. 2008;59:234-40.

38. Yu S, Varughese B, Li Z, Kushner PR. Healthcare resource waste associated with patient nonadherence and early discontinuation of traditional continuous glucose monitoring in real-world settings: a multicountry analysis. Diabetes Technol Ther. 2018;20:420-7.

39. Philipson T. Non-adherence in health care: Are patients or policy makers ill-informed? https://www.forbes.com/sites/tomasphili pson/2015/05/08/non-adherence-in-health-care-are-patients-orpolicy-makers-ill-informed/\#64939d7e4c4a. Accessed 16 July 2019. 